\title{
Partial Synchronization on Complex Networks
}

\author{
Bin Ao and Zhigang Zheng* \\ Department of Physics and the Beijing-Hong-Kong-Singapore Joint Center for Nonlinear and Complex Systems (Beijing), \\ Beijing Normal University, Beijing 100875, CHINA
}

\begin{abstract}
Network topology plays an important role in governing the collective dynamics. Partial synchronization $(\mathrm{PaS})$ on regular networks with a few non-local links is explored. Different PaS patterns out of the symmetry breaking are observed for different ways of non-local couplings. The criterion for the emergence of $\mathrm{PaS}$ is studied. The emergence of $\mathrm{PaS}$ is related to the loss of degeneration in Lyapunov exponent spectrum. Theoretical and numerical analysis indicate that non-local coupling may drastically change the dynamical feature of the network, emphasizing the important topological dependence of collective dynamics on complex networks.
\end{abstract}

PACS numbers: 05.45.Xt, 87.10.+e.

Synchronization, as a universal cooperative behavior and a fundamental mechanism in nature, has been extensively studied in relating to numerous phenomena in physics, chemistry, and biology [1, 2, 3, 4]. In recent years, there has been a growing interest in the synchronization of spatiotemporal systems, especially in synchronous dynamics on networks. Synchronization on typical complex networks, e.g., on small-world networks [ 5], scale-free networks [6, 7] or weighted network [] have been investigated recently $[\underline{9}$. In spite of these efforts, a number of fundamental questions still remain open. For example, most of previous works have been focused on the onset of global synchronization, while much less was explored for partial synchronization prior to the global case. However, there exist rich synchronous dynamics on complex networks, and many of these behaviors are closely related to partially synchronous motions. Moreover, the mechanism for synchronization on complex networks is still not clear. A good understanding of this issue should be relevant to many collective behaviors in spatiotemporal systems, especially in complex networks.

An important feature of complex networks is the existence of non-local links. On the one hand, dynamics on general complex networks (e.g., small-world or scale-free types) are usually difficult to study due to the sophisticated network topology; On the other hand, simple networks, especially regular networks, cannot be ideal models in describing real networks. Therefore, one should find some kind of network that is relatively simple but complex enough to reflect properties of typical complex networks. It is intuitive and feasible to consider a regular network plus only a few non-local links, which forms the simplest "complex" network. This can be regarded as a good bridge between regular networks and general complex networks. In this Letter, we address the issue of synchronization on rings of coupled oscillators with a few shortcut links. We reveal important partial synchronization (PaS) phenomena induced by symmetry breaking in the presence of non-local couplings. Various syn-

\footnotetext{
*Corresponding author: zgzheng@bnu.edu.cn.
}

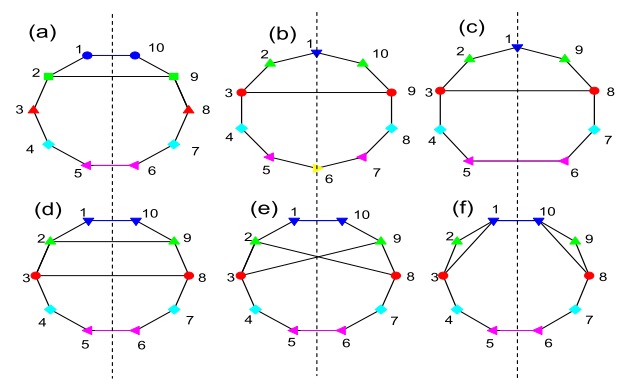

FIG. 1: Different Topologies of regular networks with nonlocal links. (a): One shortcut leads to five synchronized clusters(10 nodes); (b): One shortcut leads to six clusters (10 nodes).(c): One shortcut leads to five clusters (9 nodes). (d): Two parallel shortcuts; (e)Two crossed shortcuts; (f)The lambda-type network.

chronous patterns are observed for different types of nonlocal links. This reveals the significant role of network topology in governing the global dynamics. We analyze in detail the conditions for the emergence of $\mathrm{PaS}$ and give the criterion of the topological dependence of PaS.

We apply certain $m$-dimensional dynamics to nodes in a given network: $\dot{\vec{x}}=\vec{f}(\vec{x}), \vec{x}=\left(x_{1}, x_{2}, \cdots, x_{m}\right)$. The network is formed by using the linear coupling as edges. The dynamical equations of the network can be written as

$$
\dot{\vec{X}}=\vec{F}(\vec{X})+\varepsilon \Gamma \otimes C \vec{X},
$$

where $\vec{X}=\left(\vec{x}^{1}, \vec{x}^{2}, \cdots, \vec{x}^{N}\right), \vec{F}=\left(\vec{f}^{1}, \vec{f}^{2}, \cdots, \vec{f}^{N}\right)$, and $N$ is the number of nodes. $\varepsilon$ denotes the coupling strength, and $\Gamma: \mathbb{R}^{m} \rightarrow \mathbb{R}^{m}$ characterizes the coupling scheme among the nodes. $C=M-D$, where $M$ is the adjacency matrix of the network (the element $M_{i j}$ denotes the number of the edges that link node $i$ and $j$ ). $D$ is a diagonal matrix, and $D_{i i}=\sum_{j=1}^{N} M_{i j}$. Therefore $\sum_{j=1}^{N} C_{i j}=0$, and its largest eigenvalue $\lambda_{1}^{C}$ is 0 (we adopt $\lambda_{1} \geq \lambda_{2} \geq \cdots \geq \lambda_{N}$ throughout this Letter).

We first briefly describe the synchronous phenomena we observed in the above networks. Above all, one can 
define the average distance between two oscillators $i$ and $j$ by $d_{i j}=\lim _{T \rightarrow \infty}\left(T^{-1} \int\left\|\overrightarrow{x_{i}}-\overrightarrow{x_{j}}\right\| d t\right)$, and say they are in a synchronous state in the sense that $d_{i j} \rightarrow 0$. Denote the number of non-local links by $N_{s}$. When $N_{s}=1$, the synchronous patterns are shown in Figs. 1(a)-(c), where the nodes with the same symbol will synchronize to each other, forming several synchronous clusters. We call the formation of these synchronous clusters "partial synchronization" (PaS). Several interesting phenomena can be observed: (1) PaS emerges not only for oscillators directly linked by the non-local couplings, but also for those without shortcuts; (2) Synchronous clusters are formed by satisfying the mirror symmetry of lattices, i.e., if $(i, j)$ is a pair linked by a shortcut, then $(i+k, j-k)$ are all synchronous pairs. More interestingly, if there are two non-local links, the emergence of PaS also need not only satisfy the mirror symmetry of network, but also depend on the linking form. For example, PaS can always be achieved on networks with "parallel" links [the network with two shortcuts $(i, j)$ and $(i+k, j-k)]$, as shown in Fig. 1(d). However, for networks with "cross" shortcuts [two shortcuts $(i, j)$ and $(i+k, j+k)$ ] shown in Fig. 1(e) and networks with "lambda"-type shortcuts [two shortcuts $(i, i+k)$ and $(j, j+k)$ ] shown in Fig. 1(f), $\mathrm{PaS}$ emerges only for some choices of $k$. If $\mathrm{PaS}$ emerges on "cross" and "lambda" networks, synchronization may not emerge between pairs that are directly linked.

The above PaS behavior implies the important role of network topology in governing the collective behaviors. It is important to understand the mechanism of the above interesting PaS phenomena. For example, why does $\mathrm{PaS}$ appear on networks with mirror symmetry of sites? What is the manifestation in the dynamical exponents at the onset of PaS? Moreover, can we find a simple criterion in exactly determining whether $\mathrm{PaS}$ can appear in a given network? It is our central task to answer these important questions.

We start with theoretical analysis on the stability of complete synchronization [11]. For the synchronous state $\vec{x}^{1}(t)=\vec{x}^{2}(t)=\cdots=\vec{x}^{N}(t)=\vec{s}(t)$, by linearizing Eq. (10) near the synchronous manifold $s \overrightarrow{(t})$, i.e., $\vec{x}^{i}=\vec{s}+\vec{u}^{i}(i=$ $1,2, \cdots, N)$, one may get $\dot{\vec{U}}=\left[\vec{I}_{N} \otimes D \vec{f}+\varepsilon \Gamma \otimes C\right] \vec{U}$, where $\vec{U}=\left(\vec{u}^{1}, \vec{u}^{2}, \cdots, \vec{u}^{N}\right)$ and $D \vec{f}$ is the Jacobian function of $\vec{f}$ near the synchronous state $\vec{s}(t)$. Noticing that the first term of the new equation is block diagonal with $m \times m$ blocks, one can diagonalize $C$. Thus it becomes $\dot{\vec{v}}_{k}=$ $\left[D \vec{f}+\varepsilon \lambda_{k} \Gamma\right] \vec{v}_{k},(k=1,2, \cdots, N)$, where $\lambda_{k}$ are eigenvalues of $C$. Then Eq. (11) becomes $N$ independent blocks . To judge whether CS can emerge on a network, one needs to find the regime where the largest Lyapunov exponent of the generic equation $\dot{\vec{v}}=[D \vec{f}+\alpha \Gamma] \vec{v}$ is negative on the $\alpha$ plane ( $\alpha$ is complex). If all $\varepsilon \lambda_{k}(k=1,2, \cdots, N)$ are in the negative(stable) regime (except $\lambda_{1}=0$ ), then the CS state will be stable. Therefore, the essence of this method is to divide the system into two subsystems which dominate the dynamics on the synchronous manifold(the equation with $\lambda_{1}=0$ ) and that on the transversal mani- fold(the equation with $\left.\lambda_{i}(i=2,3, \cdots, N)\right)$, and then the emergence of the CS is determined by the stability of the transversal system.

For the case of PaS, one also needs the system to be divided into two parts, and the stability of one of them (the transverse system) determine the emergence of the PaS. On the one hand, the symmetry of a network can be defined by the invariance under a permutation transformation. Taking the case in Fig. 1(a) as an example, its symmetry requires that $F_{N} C F_{N}^{-1}=C$, where $F_{N}$ is a counteridentity matrix that satisfies $F_{i, N-i+1}=1$, for $i=1,2, \cdots, N$ and $F_{i j}=0$ for all $i+j \neq N / 2+1$, thus the network has a mirror symmetry. It is easy to verify that $\mathrm{PaS}$ is a solution of the differential equation, then each node in a cluster should possess the same dynamics 12]. Therefore the mirror symmetry is needed for the emergence of PaS. On the other hand, one can introduce new variables $\vec{W}=S \vec{X}$, where $\vec{W}=\left(\vec{w}^{1}, \vec{w}^{2}, \cdots, \vec{w}^{N}\right)$,

$$
S=\left(\begin{array}{cc}
I_{N / 2} & F_{N / 2} \\
I_{N / 2} & -F_{N / 2}
\end{array}\right)
$$

Here $I_{N / 2}$ is a $N / 2$-order identity matrix. For a network with a mirror symmetry, the above transformation can divide the system into two subsystems. Although this requires the adjacent matrix $C$ to be persymmetric (i.e., $C$ is symmetric about its anti-diagonal), the condition can always be satisfied by relabelling the node for a network with a mirror symmetry. Furthermore, if a network satisfies the mirror symmetry and its label obeys the rule in Fig. (1), the adjacent matrix $C$ can be block diagonalized by the transformation (2):

$$
G=S C S^{-1}=\left(\begin{array}{cc}
A & 0 \\
0 & B
\end{array}\right) .
$$

This transformation only re-distributes the eigenvalue spectrum of the matrix $C$ into two blocks $A$ and $B$. Since $\sum_{j=1}^{N / 2} A_{i j}=0$, the largest eigenvalue of $A$ is 0 .

By using the above transformation and considering that we only care about the dynamics near the synchronous manifold, the system can be separated into two parts, and they can be linearized near their synchronous manifold respectively:

$$
\begin{aligned}
\delta \dot{\vec{W}}_{i m} & =\left[D \vec{F}\left(\vec{W}_{i m}\right)+\varepsilon \Gamma A\right] \delta \vec{W}_{i m}, \\
\delta \dot{\vec{W}}_{t v} & =\left[D \vec{F}\left(\vec{W}_{t v}\right)+\varepsilon \Gamma B\right] \delta \vec{W}_{t v},
\end{aligned}
$$

where $\vec{W}_{i m}=\left(\vec{w}^{1}, \vec{w}^{2}, \cdots, \vec{w}^{N / 2}\right)$ and $\vec{W}_{t v}=$ $\left(\vec{w}^{N / 2+1}, \vec{w}^{N / 2+2}, \cdots, \vec{w}^{N}\right)$. For the PaS case discussed above, we have $\vec{W}_{i m}=\vec{X}_{1}+\vec{X}_{2}, \vec{W}_{t v}=\vec{X}_{1}-$ $\vec{X}_{2}$, where $\vec{X}_{1}=\left(\vec{x}^{1}, \vec{x}^{2}, \cdots, \vec{x}^{N / 2}\right)$, and $\vec{X}_{2}=$ $\left(\vec{x}^{N / 2+1}, \vec{x}^{N / 2+2}, \cdots, \vec{x}^{N}\right)$. Here, $\vec{W}_{i m}$ denotes the dynamics on synchronous manifold and $\vec{W}_{t v}$ denotes the dynamics on transversal manifold.

Back to Eqs. (3) and (4). If one increase the coupling from 0 , synchronization will be achieved for (3) and (4) 

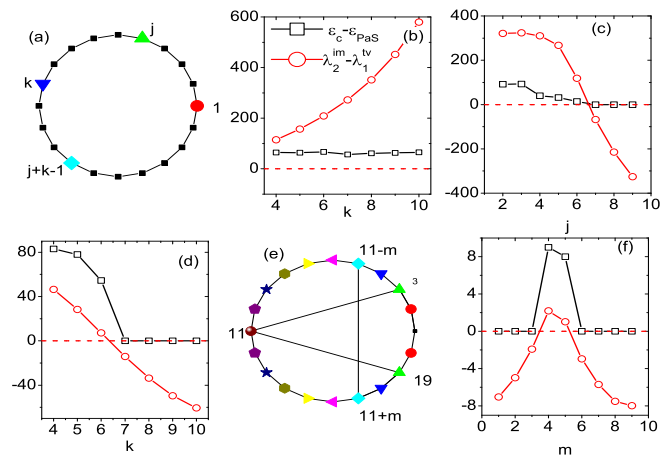

FIG. 2: (a): The label rule for $N_{s}=2$; (b)-(d): The comparison between $\varepsilon_{C}-\varepsilon_{P a S}$ and $\lambda_{2}^{i m}-\lambda_{1}^{t v}, \mathrm{~N}=20 . \lambda_{2}^{i m}-\lambda_{1}^{t v}$ are amplified by a proper times; (b): Parallel network, $\mathrm{j}=3$; (c): Cross network, $\mathrm{k}=9$; (d): Lambda network, $\mathrm{j}=3$; (e): Topology graph of the network for $N_{s}=3$; (f): The same as (b)-(d) for $N_{s}=3$.

when $\varepsilon \lambda_{2}^{i m}\left(\lambda_{2}^{i m}\right.$ is the second largest eigenvalue of $A$ ) and $\varepsilon \lambda_{1}^{t v}\left(\lambda_{1}^{t v}\right.$ is the largest eigenvalue of $B$ ) respectively fall in the stable regime of the $\alpha$ plane. The synchronization (the stability of synchronous state) of Eq. (4) suggest the emergence of PaS. If $\lambda_{2}^{i m}<\lambda_{1}^{t v}$, the synchronization of Eq.(3) will be achieved at a smaller coupling than that of Eq.(44), thus a global synchronization state has been achieved when Eq.(4) is stable. Therefore the emergence of $\mathrm{PaS}$ requires

$$
\lambda_{2}^{i m}>\lambda_{1}^{t v} .
$$

Now let us test the validity of this criterion and numerically explore the PaS phenomena. We take the Lorenz oscillator as the node of the network: $d x / d t=\sigma(y-x)$, $d y / d t=r x-y-x z, d z / d t=x y-b z$. Here $\sigma=10, r=$ $27, b=8 / 3$. We adopt the coupling scheme by the $3 \times 3$ matrix $\Gamma$ as $\Gamma_{2,1}=1$ and $\Gamma_{i, j}=0$ for $i \neq 2, j \neq 1$. Let us first try to find all possible networks that satisfy the mirror symmetry. Taking the network with two non-local links for instance (the case of $N_{c}=1$ can always topologically lead to PaS). For convenience we always take node $i=1$ as one of the ends. The second and the third ends are labelled as $j$ and $k(k>j)$, respectively. Due to the mirror symmetry, the label of the fourth end is $n_{4}=k+j-1$. There are three possible links that hold the mirror symmetry, as shown in Figs. 1(d), (e) and (f). Noticing the translational symmetry of the ring network, we have $2 \leqslant j \leqslant N / 2$ and $j \leqslant k \leqslant N / 2$. Thus by varying $j$ and $k$, all symmetric networks with two non-local couplings can be found [Fig. [2(a)].

We use $\varepsilon_{P a S}$ and $\varepsilon_{C}$ to represent the critical coupling of $\mathrm{PaS}$ and CS, respectively. Figs 2(b) and (c) give a comparison between differences $\Delta \lambda=\lambda_{2}^{i m}-\lambda_{1}^{t v}$ and $\Delta \varepsilon=\varepsilon_{C}-\varepsilon_{P a S}$ for different networks. It is instructive to find that $\Delta \lambda$ passes zero when $\Delta \varepsilon$ becomes zero. $\Delta \varepsilon \rightarrow 0$ means the coincidence between $\mathrm{CS}$ and $\mathrm{PaS}$,
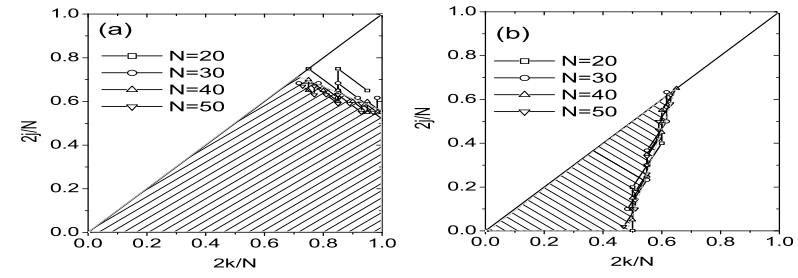

FIG. 3: The PaS regimes (labelled by sparse lines). (a): cross network; (b): lambda network.

implying that PaS doesn't appear. This good correspondence verifies our criterion in (5). Our investigations reveal that one-shortcut networks and "parallel" networks can always achieve $\mathrm{PaS}$ because both $\Delta \lambda$ and $\Delta \varepsilon$ are positive for all $k$, as shown in Fig[3 (b) for the "parallel" case. For "cross" and "lambda" networks, PaS occurs only for some configurations, as shown in Figs $3(\mathrm{c})$ and (d). To illustrate these results, we plot the PaS critical line $\Delta \lambda=0$ in the $(2 j / N, 2 k / N)$ plane. Fig 3 (a) plots the PaS regime for "cross" networks, and Fig B(b) gives the phase diagram of "lambda" networks. Different lines correspond to different nodes number. These lines coincide with each other pretty well, indicating that $\mathrm{PaS}$ depends on the topology of networks and is independent of numbers of nodes.

The above criterion for PaS can also be applied to networks with more than two non-local links, while the discussions become more complicated. However, the above phenomena and criterion still remain valid. Fig $2(\mathrm{f})$ shows one case of three shortcuts. There are 20 nodes in a network, and two non-local links are fixed to be $(11,3)$ and $(11,19)$, and the third link is $(11+m, 11-m)$, where $m=1,2, \cdots, 9$, see Fig $2(\mathrm{e})$. PaS occurs only for the $m=4,5$ cases, as shown in Fig 2 (f).

Let's observe the manifestation of the Lyapunov exponent (LE) spectrum (LES) of the network at the onset of PaS. Fig 凹(a) gives the first four largest LE's for a ring of 6 nodes without shortcuts. One can find that the third and the fourth largest LE's pass zero at the same coupling $\left(\varepsilon=\varepsilon_{C}\right)$, indicating the degeneration of LE's at the onset of CS for ring network. Fig 4 (b) is the four largest LE's of a ring network with one non-local link $(1,4)$. Obviously the presence of non-local links leads to a breaking of degeneration of LES. The fourth largest LE passes zero at a very small coupling. It also can be found that $d_{1,4}, d_{2,3}, d_{5,6}$ become 0 at the same coupling, as shown in Fig近 (c), but $d_{1,2}, d_{3,4}, d_{1,6}, d_{4,5} \neq 0$. Therefore, PaS emerges if and only if there only are two positive LE's. This conclusion was tested to be true for different node numbers and different shortcut numbers and link ways.

These results can be interpreted by analyzing eigenvalues of the adjacent matrix. The adjacent matrix of a ring network is a circulant matrix, its eigenvalues are conjugated besides the largest and the smallest ones. So when the coupling increases, $\varepsilon \lambda_{2}$ and $\varepsilon \lambda_{3}$ will fall into 

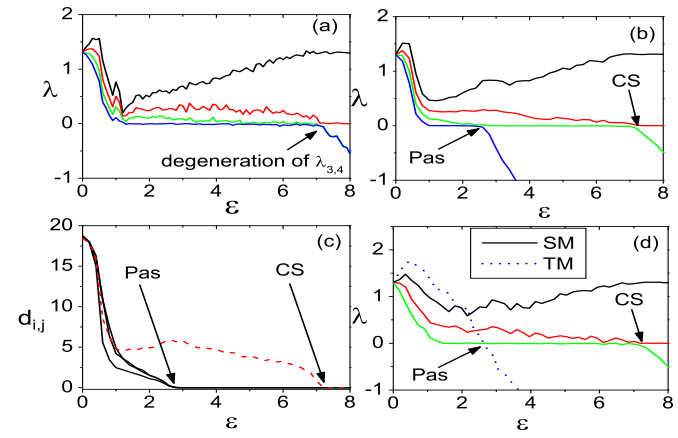

FIG. 4: (a): The first four largest LEs of a ring network; (b): The first four largest LE's of a ring network with one shortcut $(1,4) ;(\mathrm{c})$ : average trajectory distances $d_{1,4}, d_{2,3}, d_{5,6}$ (solid lines), and $d_{1,2}, d_{3,4}, d_{1,6}, d_{4,5}$ (dashed lines) varying with the coupling, where the network is the same as in (b); (d): The three largest LE's of the synchronous manifold (solid lines) and the largest LE of the transversal manifold.

the stable regime at same critical coupling, above which the third and fourth LE's corresponding to $\lambda_{2}$ and $\lambda_{3}$ become negative (there are always at least one positive LE and one zero LE for a chaotic system). When non-local couplings are applied, the degeneration in the eigenvalues and LES is lost. Since the emergence of $\mathrm{PaS}$ requires $\lambda_{2}^{i m}>\lambda_{1}^{t v}$, when $\varepsilon \lambda_{1}^{t v}$ is in stable regime, there are only two eigenvalues $\left(\lambda_{1}^{i m}=0\right.$ and $\left.\lambda_{2}^{i m}\right)$ outside of the stable regime. That is, there are only two positive LE's. One can also calculate the conditional LE spectrum of the synchronous manifold (Eq.(3)) and its corresponding transversal manifold (Eq.(4)). It can be found from
Fig 4(d) that there are only two positive conditional LE's of synchronous state(solid line) when the largest conditional LE of the transversal manifold (dotted line) passes zero.

In conclusion, in this letter we explored synchronization behaviors of complex networks with a few non-local links. The criterion for the emergence of PaS is theoretically given and verified in numerical simulations. The manifestation of Lyapunov exponent spectrum is discussed for PaS. Our results and criterion proposed in this Letter are general, and they are independent of the dynamics on sites and coupling forms. we tested different dynamics on the nodes, such as Lorenz oscillators, Rossler systems, and nonlinear maps (e.g., the logistic map and circle maps). All these numerical experiments support our criterions and conclusions. These "simple" complex networks are not only simple but also typical, and it can be a useful tool to study more complex networks. The synchronization behavior we studied in this letter reveal the importance of network topology. Different types of shortcuts may drastically change the topology and furthermore the dynamics of a network. Our work should illuminate a good step in understanding the synchronization processes on general complex networks from a dynamical point of view.

\section{Acknowledgments}

We thank Prof. Gang Hu for constructive comments and suggestions, and we are also grateful to Drs. H.Chen, Z.Cao, N.Zheng and X.Feng for very useful discussions and help.
[1] L. M. Pecora and T. L. Carroll, Phys. Rev. Lett. 64, 821(1990); A. Pikovsky, M. Rosenblum and J. Kurths, Synchronization: A universal concept in nonlinear sciences (Cambridge University Press, Cambridge, 2001); S. Boccaletti, C. Grebogi, Y. C. Lai, H. Mancini, and D.Maza, Phys. Rep. 329, 103 (2000). S. Boccaletti, J. Kurths, G. Osipov, D. L. Valladares, C. S. Zhou, Phys. Rep. 366, 1 (2002).

[2] M. G. Rosenblum, A. S. Pikovsky, and J. Kurths Phys. Rev. Lett. 76, 1804 (1996).

[3] Z. Zheng, G. Hu, and B. Hu Phys. Rev. Lett. 81, 5318 (1998). U. Ernst et al., ibid 74, 1570 (1995). G. Hu et al., ibid 85, 3377 (2000).

[4] N. Parekh, S. Parthasarathy, and S. Sinha Phys. Rev. Lett. 81, 1401 (1998).

[5] D. J. Watts and S. H. Strogatz, Nature (London) 393, 440 (1998).

[6] S.H. Strogatz, Nature 410, 268 (2001); R. Albert and A.L. Barabasi, Rev. Mod. Phys. 74, 47 (2002); M.E.J. Newman, SIAM Rev. 45, 167(2003).
[7] S. Jalan and R. E. Amritkar Phys. Rev. Lett. 90014101 (2003).

[8] D.-U. Hwang, M. Chavez et al., Phys. Rev. Lett. 94, 138701 (2005). M. Chavez, D.-U. Hwang et al., Phys. Rev. Lett. 94, 218701 (2005).

[9] M. E. J. Newman et al., Phys. Rev. Lett. 84, 3201 (2000); L. F. Lago-Fernandez et al., ibid 84, 2758 (2000); M. Barahona and L. M. Pecora, ibid 89, 054101 (2002); G. W. Wei et al., ibid 89, 284103(2002); T. Nishikawa et al., ibid 91, 014101(2003); F. M. Atay et al., ibid 92, 144101(2004); Y. Jiang et al., Phys. Rev. E 68, 065201(R)(2003); J. G. Restrepo et al., Phys. Rev. E 69, 066215(2004); Y. Moreno and A. F. Pacheco, Europhys. Lett. 68, 603(2004).

[10] I. Stewart, M Golubitsky and M. Pivato, SIAM J. Applied Dynamical Systems 2(4) 609 (2003)

[11] J. Yang et al., Phys. Rev. Lett. 80, 496 (1998); L. M. Pecora and T. L. Carroll, ibid 80, 2109 (1998).

[12] Y. Zhang et al., Phys. Rev. E 63,026211(2001). 